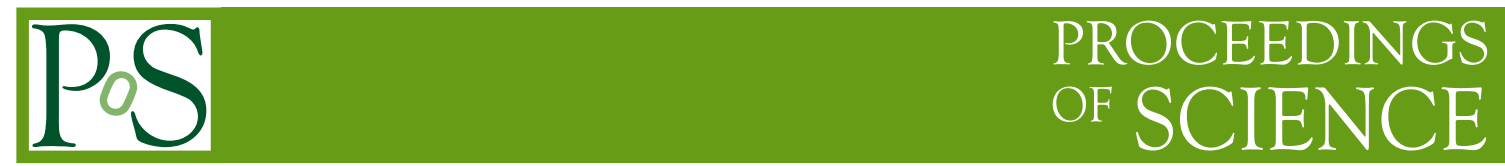

\title{
Spectroscopy, onia including exotics at $\mathrm{LHCb}$
}

\author{
Daniel Craik ${ }^{* \dagger}$ \\ University of Edinburgh \\ E-mail: dcraikecern.ch
}

\begin{abstract}
Analyses of $\mathrm{LHCb}$ data from Run I of the LHC have led to a range of new results relating to spectroscopy including the following: the first observation of the decay $D_{s 2}^{*}(2573)^{ \pm} \rightarrow D^{* \pm} K_{\mathrm{S}}^{0}$ and a measurement of its branching fraction; a Dalitz plot model of $D^{0} \rightarrow K_{\mathrm{S}}^{0} K^{ \pm} \pi^{\mp}$ decays; the discovery of pentaquarks in $\Lambda_{b}^{0} \rightarrow J / \psi p K^{-}$decays and a model-independent confirmation; and an upper limit on the production of the claimed $X(5568)^{ \pm}$tetraquark within the LHCb acceptance.
\end{abstract}

16th International Conference on B-Physics at Frontier Machines

2-6 May 2016

Marseille, France

${ }^{*}$ Speaker.

${ }^{\dagger}$ On behalf of the LHCb collaboration. 


\section{1. $D_{s}^{+}$spectroscopy}

Heavy meson spectroscopy provides essential tests used to refine models of quantum chromodynamics. Recently, such tests have been provided by studies of the $D_{s 1}^{*}(2700)^{+}, D_{s 1}^{*}(2860)^{+}$, $D_{s 3}^{*}(2860)^{+}$and $D_{s J}^{*}(3040)^{+}$mesons $[1,2,3,4,5,6]$. The former three may be interpreted as the natural spin-parity (i.e. $\left.J^{P}=0^{+}, 1^{-}, 2^{+}, \ldots\right) 2 \mathrm{~S}$ and $1 \mathrm{D}$ states of the $D_{s}^{+}$system while the latter has unnatural spin-parity (i.e. $J^{P}=0^{-}, 1^{+}, 2^{-}, \ldots$ ). No mesons that are consistent with being the three unnatural states in the $2 \mathrm{~S}$ and $1 \mathrm{D}$ levels have yet been observed. Candidates for these states should decay to the $D^{*} K$ final state.

An inclusive study of $p p \rightarrow D^{* \pm} K_{\mathrm{S}}^{0} X$ and $D^{* 0} K^{ \pm} X$ decays was performed at LHCb [7]. $D^{* \pm}$ candidates were reconstructed from the decay chains $D^{* \pm} \rightarrow D^{0} \pi^{ \pm}, D^{0} \rightarrow K^{-} \pi^{+}, K^{-} \pi^{+} \pi^{+} \pi^{-}$, and $D^{* 0}$ candidates were reconstructed from the decay chain $D^{* 0} \rightarrow D^{0} \pi^{0}, D^{0} \rightarrow K^{-} \pi^{+}$. Fits to the $D^{* \pm} K_{\mathrm{s}}^{0}, D^{* \pm} \rightarrow D^{0} \pi^{ \pm}, D^{0} \rightarrow K^{-} \pi^{+}$dataset are shown in Fig. 1, where candidates have been partitioned into two subsets that enhance the natural (NP) and unnatural (UP) contributions, respectively. Clear contributions are seen corresponding to the $D_{s 1}(2536)^{+}, D_{s 2}^{*}(2573)^{+}, D_{s 1}^{*}(2700)^{+}$and $D_{s 3}^{*}(2860)^{+}$resonances, and some evidence is seen for the $D_{S J}(3040)^{+}$state with a significance of $2.4 \sigma$. This was the first observation of the decay $D_{s 2}^{*}(2573)^{+} \rightarrow D^{*+} K_{\mathrm{S}}^{0}$ and the relative branching fraction was measured to be

$$
\frac{\mathscr{B}\left(D_{s 2}^{*}(2573)^{+} \rightarrow D^{*+} K_{\mathrm{S}}^{0}\right)}{\mathscr{B}\left(D_{s 2}^{*}(2573)^{+} \rightarrow D^{+} K_{\mathrm{S}}^{0}\right)}=0.044 \pm 0.005 \text { (stat.) } \pm 0.011 \text { (syst.) } .
$$

The helicity angle $\theta_{H}$, defined as the angle between the $K_{\mathrm{S}}^{0}$ meson and the pion from the $D^{* \pm}$ decay in the rest frame of the $D^{* \pm} K_{\mathrm{s}}^{0}$ system, yields information on the spin-parity of each resonance. Specifically, the angular distribution is expected to follow a $\sin ^{2} \theta_{H}\left(1+h \cos ^{2} \theta_{H}\right)$ distribution for an NP (UP) resonance. Fig. 2 shows the $\theta_{H}$ distributions in the regions of the $D_{s 1}^{*}(2700)^{+}$, $D_{s 3}^{*}(2860)^{+}$and $D_{s J}(3040)^{+}$resonances which are consistent with previous spin-parity assignments. The distribution in the $D_{s 3}^{*}(2860)^{+}$region is also consistent with a small UP contribution in this region.
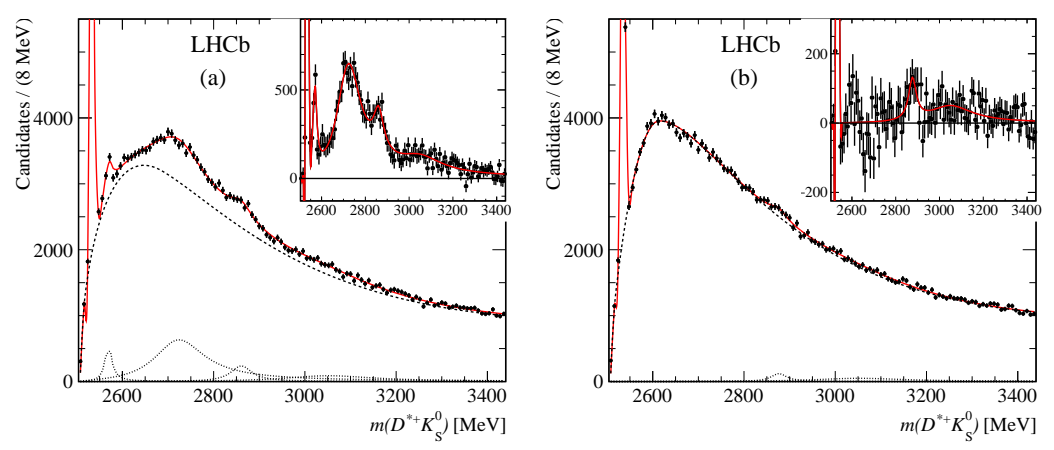

Figure 1: Distributions of the $D^{*+} K_{\mathrm{S}}^{0}$ invariant mass for the $D^{0} \rightarrow K^{-} \pi^{+}$sample with (a) $\left|\cos \theta_{H}\right|<0.5$ and (b) $>0.5$. These requirements enhance NP and UP contributions, respectively. The solid red line describes the full fitting function, the dashed line shows the background, and the dotted lines show individual resonant contributions. The insets show the same mass distributions with the backgrounds subtracted. 

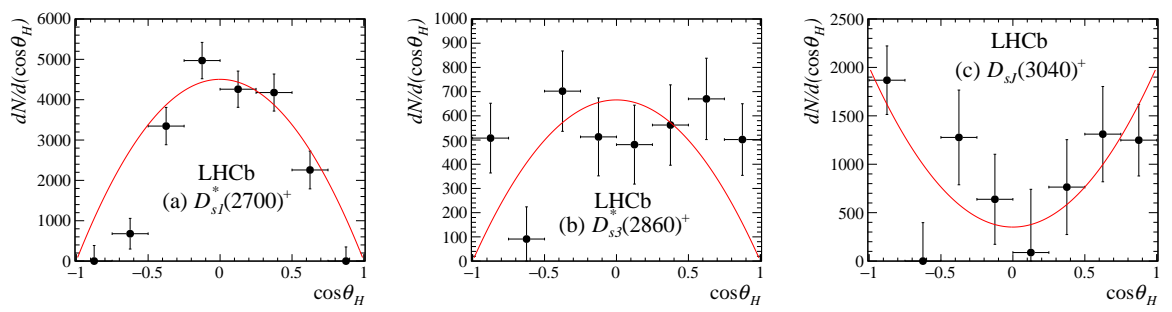

Figure 2: Distributions of the helicity angle, $\theta_{H}$, for data in the (a) $D_{s 1}^{*}(2700)^{+}$, (b) $D_{s 3}^{*}(2860)^{+}$and (c) $D_{s J}(3040)^{+}$regions with backgrounds subtracted. The angular fit to each distribution is shown as a solid red line.

\section{Dalitz plot analysis of $D^{0} \rightarrow K_{\mathrm{S}}^{0} K^{ \pm} \pi^{\mp}$ decays}

A Dalitz plot analysis of $D^{0}$ and $\bar{D}^{0}$ decays to the $K_{\mathrm{S}}^{0} K^{ \pm} \pi^{\mp}$ final states can be used to study the resonant structure and $C P$ violation present in these decays. $C P$ violation as a function of position in phase space is an important input to measurements of the CKM angle $\gamma$, while the resonant structure provides a probe of light-flavour meson spectroscopy. An analysis of these decays was performed at $\mathrm{LHCb}$ using the charge of the slow pion from the decay $D^{*+} \rightarrow D^{0} \pi_{\text {slow }}^{+}$to tag the initial flavour of the decaying meson. The amplitude model describing each Dalitz plot is constructed using the isobar formalism

$$
\mathscr{M}_{K_{\mathrm{S}}^{0} K^{ \pm} \pi^{\mp}}\left(m_{K_{\mathrm{S}}^{0} \pi^{ \pm}}, m_{K^{\mp} \pi^{ \pm}}\right)=\sum_{R} a_{R} e^{i \phi_{R}} \mathscr{M}_{R}\left(m_{K_{\mathrm{S}}^{0} \pi^{ \pm}}, m_{K^{\mp} \pi^{ \pm}}\right)
$$

where $\mathscr{M}_{R}$ describes the resonant or nonresonant contribution, $R$, and $a_{R} e^{i \phi_{R}}$ is a complex coefficient determined from the fit. Projections of the fits to both Dalitz plots are shown in Fig. 3. The model significantly favours the inclusion of percent-level contributions from the $\rho(1450)^{ \pm}$and $\rho(1700)^{ \pm}$resonances, which were previously observed in Ref [9] but are not well established.
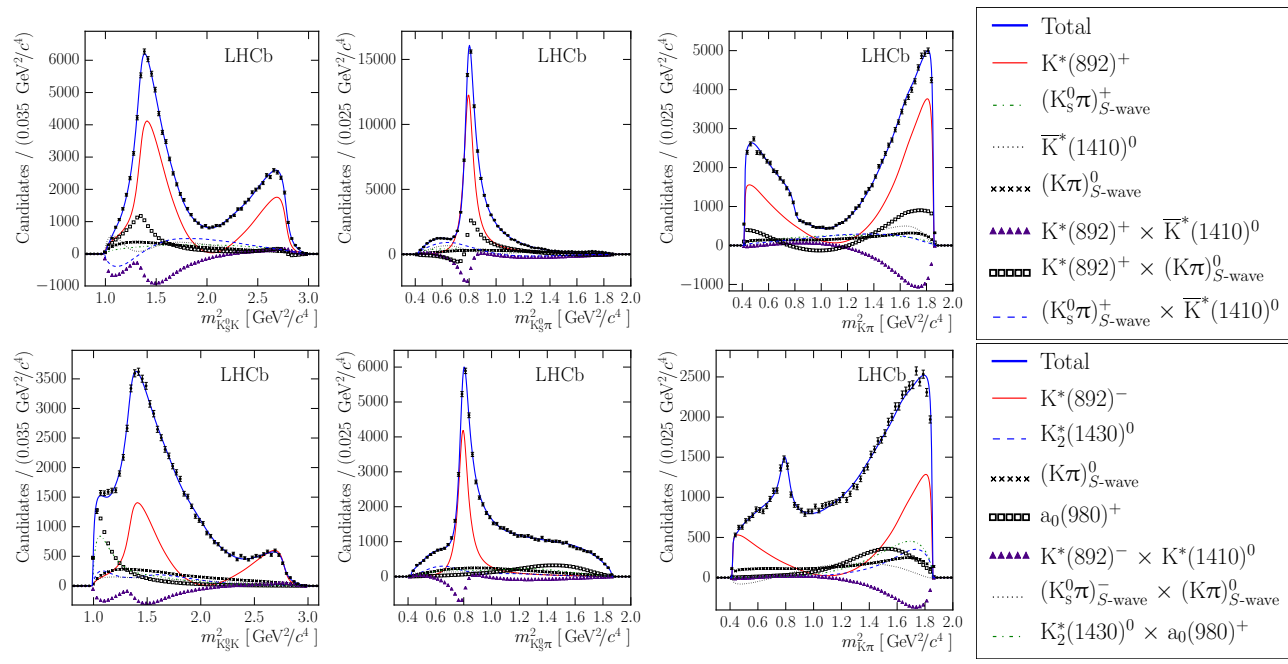

Figure 3: Invariant mass projections of the (top) $K_{\mathrm{S}}^{0} K^{-} \pi^{+}$and (bottom) $K_{\mathrm{S}}^{0} K^{+} \pi^{-}$Dalitz plots: (left) $m\left(K_{\mathrm{S}}^{0} K^{ \pm}\right)$, (centre) $m\left(K_{\mathrm{S}}^{0} \pi^{\mp}\right)$ and (right) $m\left(K^{\mp} \pi^{\mp}\right)$. The fit components are as described in the legends. 
The fit results are consistent with $C P$ conservation, with a $p$-value of 0.54 . The branching ratio was measured across the full phase space and also in the region of the $K^{*}(892)^{ \pm}$resonance

$$
\begin{gathered}
\frac{\mathscr{B}\left(D^{0} \rightarrow K_{\mathrm{S}}^{0} K^{+} \pi^{-}\right)}{\mathscr{B}\left(D^{0} \rightarrow K_{\mathrm{S}}^{0} K^{-} \pi^{+}\right)}=0.655 \pm 0.004 \text { (stat.) } \pm 0.006 \text { (syst.) } \\
\frac{\mathscr{B}\left(D^{0} \rightarrow K^{*-} K^{+}\right)}{\mathscr{B}\left(D^{0} \rightarrow K^{*+} K^{-}\right)}=0.370 \pm 0.003 \text { (stat.) } \pm 0.012 \text { (syst.), }
\end{gathered}
$$

and the corresponding coherence factors are measured to be

$$
R_{K_{\mathrm{S}}^{0} K \pi}=0.573 \pm 0.007 \pm 0.019, \quad R_{K^{*} K}=0.831 \pm 0.004 \pm 0.010 .
$$

\section{Pentaquark contributions in $\Lambda_{b}^{0} \rightarrow J / \psi p K^{-}$decays}

An LHCb study of $\Lambda_{b}^{0} \rightarrow J / \psi p K^{-}$decays led to the first observation of two new resonant states, labelled $P_{c}(4450)^{+}$and $P_{c}(4380)^{+}$, which decay to the $J / \psi p$ final state and are consistent with being pentaquarks. Using a $6 \mathrm{D}$ amplitude analysis, both pentaquarks were found to be necessary to successfully fit the data with significances in excess of $9 \sigma$ [10]. In addition to these exotic contributions, the three-body decay involves many contributions from resonances in $m\left(p K^{-}\right)$. As the spectrum of these $p K^{-}$states is poorly understood (see Fig. 4(a)), a 2D model-independent study has now been performed to test the hypothesis that structures in the $p K^{-}$system could fake the observed pentaquark signals [11]. The analysis is performed in bins in $m\left(p K^{-}\right)$with the angular distribution in each bin described by a sum of Legendre polynomials,

$$
\frac{d N}{d \cos \theta_{\Lambda^{*}}}=\sum_{l=0}^{l_{\max }}\left\langle P_{l}^{U}\right\rangle P_{l}\left(\cos \theta_{\Lambda^{*}}\right)
$$

where $N$ is the efficiency-corrected and background-subtracted yield, $\theta_{\Lambda^{*}}$ is the angle between the proton and the $J / \psi$ meson in the rest frame of the $p K^{-}$system, $P_{l}$ is the $l$ th Legendre polynomial, $l_{\max }$ is the highest-order term uesd in the summation and $\left\langle P_{l}^{U}\right\rangle$ is the Legendre moment of rank $l$,

$$
\left\langle P_{l}^{U}\right\rangle=\int_{-1}^{+1} d \cos \theta_{\Lambda^{*}} P_{l}\left(\cos \theta_{\Lambda^{*}}\right) d N / d \cos \theta_{\Lambda^{*}}
$$

As a resonance of spin $J$ in the $p K^{-}$system may only contribute to moments up to $2 J$ then, in the absence of exotic states, the angular distribution should remain well described when the sum over $l$ is truncated. Conversely, exotic contributions in either the $J / \psi p$ or $J / \psi K^{-}$system would introduce non-zero contributions to higher moments. The moments expected within each mass bin, and hence the values of $l_{\max }$, were determined based on theoretical predictions and observed resonances as shown in Fig. 4(a).

To test for exotic contributions, a likelihood ratio is constructed between a null hypothesis in which only contributions up to $l_{\max }$ were included and an alternative hypothesis with higher moments also included. As shown in Fig. 4(b), the likelihood ratio determined from data is significantly separated from the value determined from simulated data containing only $p K^{-}$contributions and consistent with the value from simulated data containing two pentaquark resonances. The $m(J / \psi p)$ distribution of data is shown in Fig. 4(c) with the results from both hypotheses superimposed. 

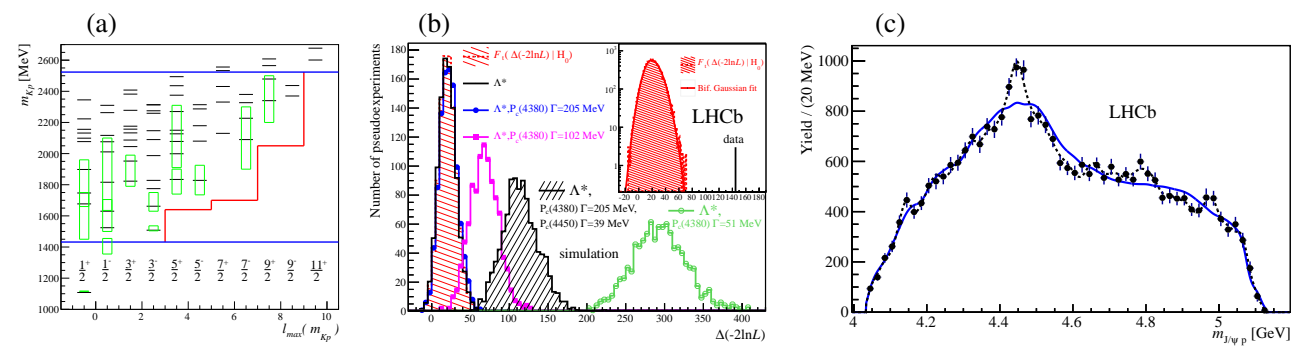

Figure 4: (a) Masses of (green) measured and (black) predicted $\Lambda^{*}$ states. The red line shows $l_{\max }$ as a function of mass and the blue lines show the kinematic limits of $m\left(p K^{-}\right)$in the three-body decay. (b) The difference in log likelihood determined from (red hatched) model-independent pseudoexperiments and (as labelled) various amplitude models. (c) The distribution of data in $m(J / \psi p)$ with the results from (blue) the null hypothesis and (black dashed) the alternative hypothesis superimposed.

\section{4. $B_{s}^{0} \pi^{ \pm}$spectroscopy}

The D0 collaboration has recently announced evidence for a new tetraquark, labelled $X(5568)^{ \pm}$, decaying to the $B_{s}^{0} \pi^{ \pm}$final state [12]. Within the D0 acceptance, the production rate of this state was measured relative to the total $B_{s}^{0}$ production rate to be $\rho_{X}^{\mathrm{D} 0}=(8.6 \pm 1.9 \pm 1.4) \%$. An LHCb study was performed to search for this state and measure its production within the LHCb acceptance [13]. Large clean samples of $B_{s}^{0}$ candidates were reconstructed from the $J / \psi \phi$ and $D_{s}^{-} \pi^{+}$ final states and combined with pion candidates. Fig. 5 shows the distribution of $m\left(B_{s}^{0} \pi^{ \pm}\right)$compared to the signal that would be expected for $\rho_{X}^{\mathrm{LHCb}}=8.6 \%$. The study found no evidence for the claimed state. Within the $\mathrm{LHCb}$ acceptance, limits are set on the production rate of $0.9 \%(1.0 \%)$ at the $90 \%$ (95\%) confidence level for $B_{s}^{0}$ mesons with $p_{\mathrm{T}}>5 \mathrm{GeV} / c$ and $1.6 \%(1.8 \%)$ for mesons with $p_{\mathrm{T}}>10 \mathrm{GeV} / c$.

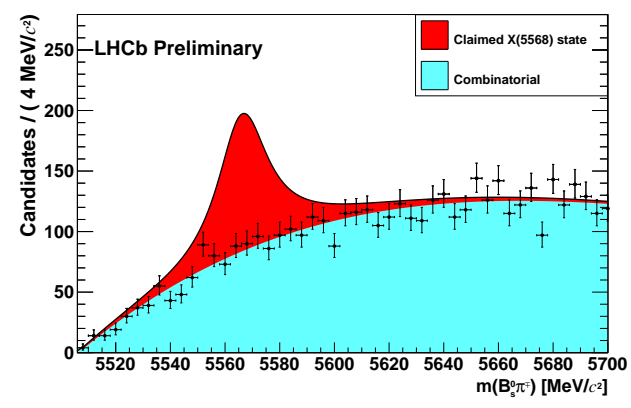

Figure 5: Distribution of $m\left(B_{s}^{0} \pi^{ \pm}\right)$with $p_{\mathrm{T}}\left(B_{s}^{0}\right)>10 \mathrm{GeV} / c$. A hypothetical signal component corresponding to $\rho_{X}^{\mathrm{LHCb}}=8.6 \%$ is superimposed.

\section{Summary}

Analyses of LHCb data from Run I of the LHC continue to produce new meson spectroscopy results and drive the search for new exotic states $[14,15,16]$. With Run II of the LHC ongoing, more exciting results are expected over the next few years. 


\section{Acknowledgements}

The author thanks the United Kingdom's Science and Technology Facilities Council.

\section{References}

[1] B. Aubert et al. (BaBar collaboration), Observation of a new $D_{s}$ meson decaying to DK at a mass of 2.86GeV/c2 , Phys. Rev. Lett. 97 (2006) 222001 [hep-ex/0607082].

[2] J. Brodzicka et al. (Belle collaboration), Observation of a new $D_{s J}$ meson in $B^{+} \rightarrow \bar{D}^{0} D^{0} K^{+}$decays, Phys. Rev. Lett. 100 (2007) 092001 [hep-ex/ 0707.3491$].$

[3] R. Aaij et al. (LHCb collaboration), Study of $D_{s J}$ decays to $D^{+} K_{S}^{0}$ and $D^{0} K^{+}$final states in $p p$ collisions, JHEP 10 (2012) 151 [hep-ex/1207.6016].

[4] R. Aaij, et al. (LHCb collaboration), Observation of overlapping spin-1 and spin-3 $\bar{D}^{0} K^{-}$resonances at mass $2.86 \mathrm{GeV/c}{ }^{2}$, Phys. Rev. Lett. 113 (2014) 162001 [hep-ex/1407. 7574].

[5] R. Aaij et al. (LHCb collaboration), Dalitz plot analysis of $B_{s}^{0} \rightarrow \bar{D}^{0} K^{-} \pi^{+}$decays, Phys. Rev. D90 (2014) 072003 [hep-ex/1407. 7712].

[6] B. Aubert et al. (BaBar collaboration), Study of $D_{s J}$ decays to $D^{*} K$ in inclusive $e^{+} e^{-}$interactions, Phys. Rev. D80 (2009) 092003 [hep-ex/ 0908 . 0806].

[7] R. Aaij et al. (LHCb collaboration), Study of $D_{s J}^{+}$mesons decaying to $D^{*+} K_{S}^{0}$ and $D^{* 0} K^{+}$final states, JHEP 02 (2016) 133 [hep-ex/1601.01495].

[8] R. Aaij et al. (LHCb collaboration), Studies of the resonance structure in $D^{0} \rightarrow K_{S}^{0} K^{ \pm} \pi^{\mp}$ decays, Phys. Rev. D93 (2016) 052018 [hep-ex/1509.06628].

[9] M. Bargiotti et al. (OBELIX collaboration), Coupled channel analysis of $\pi^{+} \pi^{-} \pi^{0}, K^{+} K^{-} \pi^{0}$ and $K^{ \pm} K_{\mathrm{S}}^{0} \pi^{\mp}$ from $\bar{p} p$ annihilation at rest in hydrogen targets at three densities, Eur. Phys. J. C26 (2003) 371 .

[10] R. Aaij et al. (LHCb collaboration), Observation of $J / \psi$ p resonances consistent with pentaquark states in $\Lambda_{b}^{0} \rightarrow J / \psi p K^{-}$decays, Phys. Rev. Lett. 115 (2015) 072001 [hep-ex/1507.03414].

[11] R. Aaij et al. (LHCb collaboration), Model-independent evidence for $J / \psi p$ contributions to $\Lambda_{b} \rightarrow J / \psi p K^{-}$decays, submitted to Phys. Rev. Lett. [hep-ex/1604.05708].

[12] V. M. Abazov et al. (D0 collaboration), Evidence for a $B_{s}^{0} \pi^{ \pm}$state, submitted to Phys. Rev. D [hep-ph/1602.07588].

[13] R. Aaij et al. (LHCb collaboration), Search for structure in the $B_{s}^{0} \pi^{ \pm}$invariant mass spectrum, LHCb-CONF-2016-004 (2016).

[14] R. Aaij et al. (LHCb collaboration), Evidence for exotic hadron contributions to $\Lambda_{b}^{0} \rightarrow J / \psi p \pi^{-}$ decays, submitted to Phys. Rev. Lett. [hep-ex/1606.06999].

[15] R. Aaij et al. (LHCb collaboration), Observation of $J / \psi \phi$ structures consistent with exotic states from amplitude analysis of $B^{+} \rightarrow J / \psi \phi K^{+}$decays, submitted to Phys. Rev. Lett. [hep-ex/1606.07895].

[16] R. Aaij et al. (LHCb collaboration), Amplitude analysis of $B^{+} \rightarrow J / \psi \phi K^{+}$decays, submitted to Phys. Rev. D [hep-ex/1606.07898]. 